KfK 4071

April 1986

\title{
Charge Radii and Moments of Tin Nuclei by Laser Spectroscopy
}

M. Anselment, K. Bekk, A. Hanser, H. Hoeffgen,

G. Meisel, S. Göring, H. Rebel, G. Schatz Institut für Kernphysik

\section{Kernforschungszentrum Karlsruhe}




\section{KERNFORSCHUNGSZENTRUM KARLSRUHE}

Institut für Kernphysik

KfK 4071

CHARGE RADII AND MOMENTS OF TIN NUCLEI BY LASER SPECTROSCOPY

M.Anselment,* K.Bekk, A.Hanser, H.Hoeffgen, ** G.Meisel, S.Göring, H. Rebel, and G.Schatz

*Present address: Dep. of Physics and Astronomy, Louisiana State Univ., Baton Rouge, Louisiana, U.S.A.

**Present address: Batelle-Institut e.V., D-6000 Frankfurt/Main 90, Fed. Rep. Germany.

Kernforschungszentrum Karlsruhe GmbH, Karlsruhe 
Als Manuskript vervielfältigt

Für diesen Bericht behalten wir uns alle Rechte vor

Kernforschungszentrum Karisruhe $\mathrm{GmbH}$

Postfach 3640, 7500 Karlsruhe 1

ISSN 0303-4003 


\begin{abstract}
The isotope shift and hyperfine structure of the optical $\mathrm{Sn} I$ resonance transition $5 p^{2}{ }^{3} P_{0} \rightarrow 5 p 6 s{ }^{3} P_{1}$ at $\lambda=286.3 n m$ have been studied for $18 \mathrm{Sn}$ nuclei including 2 isomers. Laser induced resonance fluorescence from a collimated atomic beam of tin was observed using a tunable $\mathrm{cw}$ dye laser with frequency doubler. The electromagnetic nuclear moments and changes of the mean square charge radii of the nuclear charge distributions were determined. The results are discussed with respect to the information they provide on the nuclear structure of the nuclei investigated; they are compared with various theoretical models.
\end{abstract}

DIE LASERSPEKTROSKOPISCHE BESTIMMUNG DER KERN-LADUNGSRADIEN UND -MOMENTE VON ZINN-ATOMKERNEN

\title{
Zusammenfassung
}

Die Isotopieverschiebung und die Hyperfeinaufspaltung des optischen Sn I-Überganges $5 p^{2}{ }^{3} P_{0} \rightarrow 5 p 6 s{ }^{3} P_{1}$ bei $\lambda=286.3 n m$ wurden für 18 Zinnkerne, darunter 2 Isomere, untersucht. Dazu wurde die laserinduzierte Fluoreszenz an einem kollimierten Atomstrahl beobachtet; als Lichtquelle wurde ein durchtimmbarer Dauerstrich-Farbstofflaser mit Frequenzverdoppler verwendet. Es wurden die elektromagnetischen Kernmomente und die Änderungen der mittleren quadratischen Ladungsradien der Kernladungsverteilung bestimmt. Die Ergebnisse werden im Hinblick auf die Kernstruktur der untersuchten Atomkerne diskutiert und mit verschiedenen theoretischen Modellen verglichen. 


\section{Introduction}

The energies of atomic transitions, in particular those associated with s electrons, alter slightly with the neutron number of the atomic nucleus. This isotope shift arises from the difference of the nuclear masses of the isotopes and from the difference of the nuclear Coulomb field experienced by the atomic electrons involved in the transition. The latter contribution, historically referred to as volume effect, ${ }^{1}$ is due to isotopic differences of the nuclear charge distributions of which the mean square charge radius $\left\langle r^{2}\right\rangle$ enters into the volume effect calculation. Effects of nuclear polarization associated with the interaction between atomic and nuclear states also give rise to isotope shifts, but this contribution is expected to be so small that it can be ignored for transitions of normal atoms. ${ }^{2}$ In addition, the interaction of the nuclear magnetism and of nonspherical components of the nuclear charge distribution with the atomic electrons lead to the well known hyperfine splitting of atomic lines.

Studies of these nuclear effects observed in atomic transitions are an important source of nuclear structure information, in particular about the systematics of nuclear charge radii and electromagnetic moments in long isotopic series. The renewed interest $^{-5}$ in nuclear structure investigations of this kind originates from the progress of experimental techniques, mainly due to the application of laser spectroscopic methods ${ }^{6}$ which give enhanced resolution, improved accuracy and higher sensitivity. In parallel, refined theoretical descriptions of the experimental findings have been developed on the basis of macroscopic ${ }^{7-9}$ and microscopic ${ }^{10-13}$ structure models accounting even for details in the trend of the nuclear charge radii. The observed trends are globally fairly well desribed by the droplet model, ${ }^{7}$ recently extended by the inclusion of deformation effects ${ }^{14}$ which are clearly required by the experimental data (see Ref. 15), e.g. revealing the onset of deformation in new nuclidic regions far from stability. ${ }^{16}$ In the neighborhood of magic nuclei (as, e.g., lead ${ }^{1718}$ ), where effects of static or dynamic deformations are of reduced importance, there are features (and accordingly deviations from the droplet model) which originate from the specific shell structure ${ }^{18} 20,12$ and may shed some light on pairing interactions. ${ }^{13}$

The present paper reports on laser spectroscopic measurements of the

isotope shift and the hyperfine splitting in the $5 p^{2}{ }^{3} \mathrm{P}_{0} \rightarrow 5 p 6 s{ }^{3} \mathrm{P}_{1}$ 
resonance transition at $\lambda=286.3 \mathrm{~nm}$ of stable and radioactive $S n$ atoms, providing results on a total of 18 nuclei including 2 isomers. The experiments extend earlier measurements on stable isotopes ${ }^{21-23}$ and rf studies of radioactive ${ }^{113} \mathrm{Sn}$ and ${ }^{121} \mathrm{Sn}$; the latter gave results for the values of the nuclear ground state spins and of the electromagnetic moments, though without sign. ${ }^{24}$ Recently, the $5 p{ }^{2}{ }^{1} S_{0} \rightarrow 5 p 6 s{ }^{1} P_{1}$ transition $(\lambda=452.5 \mathrm{~nm})$ has been investigated ${ }^{20}$ by collinear laser spectroscopy extending our findings to ${ }^{109} \mathrm{Sn}$ and ${ }^{108} \mathrm{Sn}$.

The specific interest in Sn (which has the highest number of stable isotopes among all elements) arises from the changing polarization of the closed proton shell $(Z=50)$ with the variation of the neutron number between $N=50$ (i.e. from the experimentally undiscovered doubly magic ${ }^{100} \mathrm{Sn}$ ) to $\mathrm{N}=82$ (i.e. the doubly magic ${ }^{132} \mathrm{Sn}$ ). In the corresponding case of $\mathrm{Ca}$ the polarization of the proton core is manifested by a rather peculiar variation of the charge radii, 25 showing decreasing values of the mean square charge radii $\left\langle r^{2}\right\rangle$ charge when ${ }^{48} \mathrm{Ca}$ is approached. The corresponding ${ }^{132} \mathrm{Sn}$ is inaccessible by present laser spectroscopic methods. From the point of view of the single particle model, however, a subshell closure is expected at $N=64$. Effects of such subshell closure are also reported for the charge radii of the neighboring element $\mathrm{Cd} .^{26}$

\section{Experimental arrrangement}

The experimental method applied is laser-induced fluorescence spectroscopy, i.e. free tin atoms, obtained by vacuum evaporation of elementary tin samples, are irradiated with resonant laser light to excite them (see Fig. 1). The resonance absorption is detected indirectly by monitoring the spontaneously reemitted fluorescence light. For the light absorption the resonance transition from the ground state $5 s^{2} 5 p^{2}{ }^{3} P_{0}$ to the $5 s^{2} 5 p 6 s^{3} \mathrm{P}_{1}$ excited state requiring a wavelength $\lambda=286.3 \mathrm{~nm}$ is used. For efficient detection the decay into the $5 s^{2} 5 p^{2}{ }^{3} D_{2}$ level at $\lambda=380.1 \mathrm{~nm}$ was chosen. 27,28 The experimental setup has been described elsewhere, ${ }^{17}$ we therefore restrict the description mainly to special features and alterations that were introduced for the tin experiments.

The evaporation of the tin samples required temperatures of about $2100 \cdot \mathrm{K}$ which are much higher than those used previously in the apparatus. This temperature is considerably higher than that one required for 


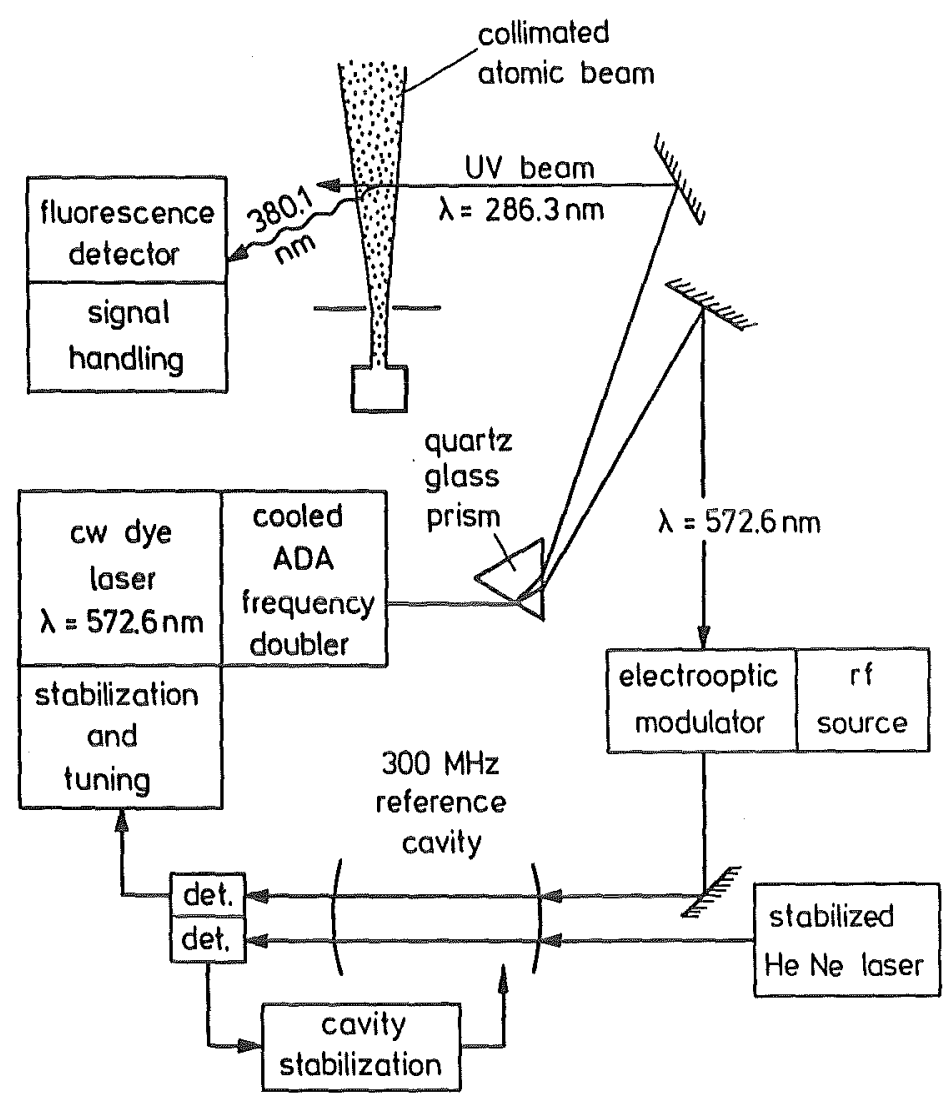

FIG. 1. Schematic diagram of the experimental arrangement.

evaporation of bulk tin. The reason possibly is that the samples are so small that the tin atoms are spread over the crucible in low concentration which seems to bind them more strongly to its surface. Therefore a new heater of the electron bombardment type was developed. The anode is a vertically mounted tube made of high purity graphite. The crucibles are made of spectroscopically pure graphite so that practically no tin was detected with an empty crucible heated in the oven. The fluorescence detector was carefully shielded so that the stray light from the hot oven was reduced to a tolerable amount of $50 \mathrm{~Hz}$ count rate at operating conditions. It was of particular importance to construct the oven for long service intervals since it required a special handling procedure to repair it because of the radioactive contamination after use. The electrical heating power ( $2000 \mathrm{~V}$; current up to $700 \mathrm{~mA}$ ) is automatically stabilized by control of the cathode heater current to correct for anode to cathode backheating and other changes of the electrical characteristics. The atomic beam is collimated by an aperture in the crucible cover and an adjustable slit for a residual Doppler width that gives an observed full line width between 40 and $70 \mathrm{MHz}$. The natural width is $32 \mathrm{MHz} .^{22}$ Small samples are investigated with the slit fully open to gain in signal strength; the line width then was $70 \mathrm{MHz}$. 
About $30 \%$ of the $380.1 \mathrm{~nm}$ fluorescence light is collected and focussed onto the bialkali photocathode of a type 9635 B photomultiplier by EMI. An interference filter with about $8 \mathrm{~nm}$ half width and $20 \%$ transmission is used to suppress stray light from different sources. The overall detection efficiency of the system including the atomic beam collimation losses is about one signal photon count per $2 \cdot 10^{5}$ sample atoms. $100 \mathrm{pg}$ samples $\left(5 \cdot 10^{11}\right.$ atoms) gave a signal peak count rate of 2 $\mathrm{kHz}$ lasting for about $10 \mathrm{sec}$. The smallest sample size for useful signals was $150 \mathrm{pg}$.

The cW dye laser used was a model CR 699-21 by Coherent with Rhodamine $6 G$ dye dissolved in ethylene glycol and pumped by an argon ion laser. The single mode output power at $572.6 \mathrm{~nm}$ typically was $1.5 \mathrm{~W}$ for 7 to $9 \mathrm{~W}$ of single line $(514.5 \mathrm{~nm})$ pump power. To generate the 286.3 $\mathrm{nm}$ UV light required the dye laser radiation was frequency doubled in a cooled ADA crystal. ${ }^{17}$ The UV power obtained from $1.5 \mathrm{~W}$ of dye laser light was about $1.2 \mathrm{~mW}$ which was sufficient to fully saturate the $\mathrm{Sn}$ transition. The fundamental visible and the harmonic UV light were separated by a quartz glass prism. All frequency measurements as well as frequency controls were done using the fundamental beam.

Two different dye laser tuning schemes were used. If a new isotope with unknown hyperfine structure was investigated the laser was operated with its internal scan while the photon counts were accumulated in parallel by a multichannel counter. Thus rough line positions were found. To correct the results from such search runs for the scan nonlinearity the equidistant resonances of a confocal $300 \mathrm{MHz}$ resonator were recorded simultaneously.

In subsequent measurements the positions of all components were determined more accurately; the dye laser was then tuned under rf control. In this series of experiments one radioactive tin sample served to measure one component only. To make best use of the limited signal duration, the laser was first scanned in a narrow range only over this component. After the sample was exhausted the empty crucible was replaced with one that contained a reference isotope. To achieve the long term stability required for this procedure as well as the accuracy desired, rf laser tuning was employed. Instead of using the heterodyne technique as before, ${ }^{17}$ a different $r f$ method was applied that requires only one dye laser instead of two and which is superior in accuracy as compared to the earlier setup. The method ${ }^{2-31}$ is based on $r f$ generated 
optical sidebands (see Fig. 1), which are generated by amplitude modulating the dye laser beam with a well known of frequency; it defines the separation between the laser or carrier frequency and the side frequencies. An electro-optic modulator (model 1080 by Lasermetrics) is used for this purpose; it is driven by the output of a microwave synthesizer.

One of the sidebands is locked to an optical resonator with a free spectral range (FSR) of $300 \mathrm{MHz}$ which is fixed in frequency by an iodine stabilized HeNe laser. The sideband lock is accomplished by a feedback to the dye laser, i.e. the laser frequency is controlled such that the sideband chosen is held on the optical resonator peak. The peak lock is realized in the usual way by dithering the etalon at a rate of 4.6 $\mathrm{kHz}$ combined with phase sensitive detection. Any frequency change of the driving of oscillation causes a dye laser frequency shift by precisely the same amount thus defining the frequency scale. The rf synthesizer is operated under computer control; the laser is tuned in discrete steps of 5 or $10 \mathrm{MHz}$ width across the resonance to be recorded. To speed up tuning and to reduce residual errors the computer generates an analog feed-forward voltage that closely matches the expected control signal. In this way a tuning range of $1 \mathrm{GHz}$ is accessible which was only limited by the synthesizer and rf power amplifier available. Since the dye laser light is frequency doubled, the corresponding tuning range on UV or atomic frequency level in this experiment is $2 \mathrm{GHz}$. The tuning range can be further doubled by locking the high or low frequency sideband to the reference resonator in turn.

To make full use of the scanning range sometimes a $2 \mathrm{GHz}$ confocal etalon was placed in front of the $300 \mathrm{MHz}$ reference cavity to filter the incoming light: The etalon serves to suppress the carrier and the unwanted sideband and to transmit the sideband used for locking. In this way undesired transmission coincidences with respect to the reference cavity are avoided that otherwise occur when the modulation frequency is a multiple of FSR/2 $=150 \mathrm{MHz}$. To facilitate locking the 2 $\mathrm{GHz}$ filter to the desired sideband the sidebands are marked by a frequency modulation ( $4 \mathrm{MHz}$ width at a speed of several $\mathrm{kHz}$ ) applied to the modulating $r f .{ }^{31}$ The filter lock utilizes a phase sensitive detection scheme which allows to distinguish (i) the unmodulated carrier from the modulated side frequencies and (ii) the two sidebands from each other since their frequency modulation is opposite in phase. 
For the highly accurate measurements of stable isotopes, however, the extra $2 \mathrm{GHz}$ filter was not used to reduce the complexity of the system. The coincidences were avoided in this operating mode by choosing the rf tuning ranges appropriately; this can be accomplished making use of the dispersion of the gas inside the reference etalon housing by changing its pressure slightly.

\section{Sample preparation}

The samples of different isotopes were supplied in different ways. ${ }^{113} \mathrm{Sn}$ could be purchased since it is a relatively long-lived isotope. $121,121 \mathrm{~m}, 123,125 \mathrm{Sn}$ were made from enriched stable $\mathrm{Sn}$ isotopes by neutron activation in the thermal flux of the two reactors of the Kernforschungsanlage at Jülich. For the neutron deficient isotopes $110,111_{\mathrm{Sn}}$ and the isomers $117 \mathrm{~m}, 121 \mathrm{~m}_{\mathrm{Sn}}$, targets of cadmium were irradiated with 10 MeV alpha particles using the internal beam of the Karlsruhe Isochronous Cyclotron.

After production the samples needed purification. This was particularly true for the reactor-made isotopes; this material underwent mass separation to concentrate the nuclei of interest. Thus typically 200 $\mathrm{pg}$ of artificial tin were extracted from $20 \mathrm{mg}$ of bulk material; the remaining stable $\mathrm{Sn}$ impurity content was about $50 \mathrm{ng}$. When $\mathrm{Sn}$ was made from cadmium, vacuum distillation was used. At a temperature of about $600 \mathrm{~K}$ the Cd target of about $30 \mathrm{mg}$ is vaporized leaving most of the Sn content back in the crucible. No mass separation was done with these samples since the loss is about $90 \%$. This however leaves the tin impurity of the target material back in the sample. Therefore in the case of ${ }^{111} \mathrm{Sn}$ the ${ }^{112} \mathrm{Cd}$ target material used was vacuum distilled before irradiation to reduce the tin impurity. By the handling, however, the targets again were contaminated with several ng of natural $\mathrm{Sn}$.

\section{Measurements}

The experiments were done in two steps: First a resonance search for a new isotope was performed which gave a coarse value of the component positions; the internal laser scanning mode was used for these records. Fig. 2 is a record of such scans for ${ }^{111} \mathrm{Sn}$. Two of the three ${ }^{111} \mathrm{sn}$ components are included in the scan range. The natural $\mathrm{Sn}$ contamination is visible as ${ }^{117,119} \mathrm{Sn}$. All of the ${ }^{113,110} \mathrm{Sn}$ and most of the stable ${ }^{112} \mathrm{Sn}$ is artificial since it is produced together with the ${ }^{111} \mathrm{Sn}$. 
Most of the resonances thereafter were determined more accurately in a second measurement with of sideband tuning. The radioactive samples were measured with a linewidth of $70 \mathrm{MHz}$ and frequency steps of 10 $\mathrm{MHz}$. The signal count rates were 1 to $5 \mathrm{kHz}$, the counting time then was chosen to be $150 \mathrm{msec}$ per point. Background counts resulted mainly from scattered oven light $(50 \mathrm{~Hz}$ at the full temperature of $2100 \mathrm{~K}$ ) plus a rate of less than $70 \mathrm{~Hz}$ from the radioactivity. The stable isotopes were investigated at a width of $40 \mathrm{MHz}$; the count rates ranged from 10 to 700 $\mathrm{kHz}$.

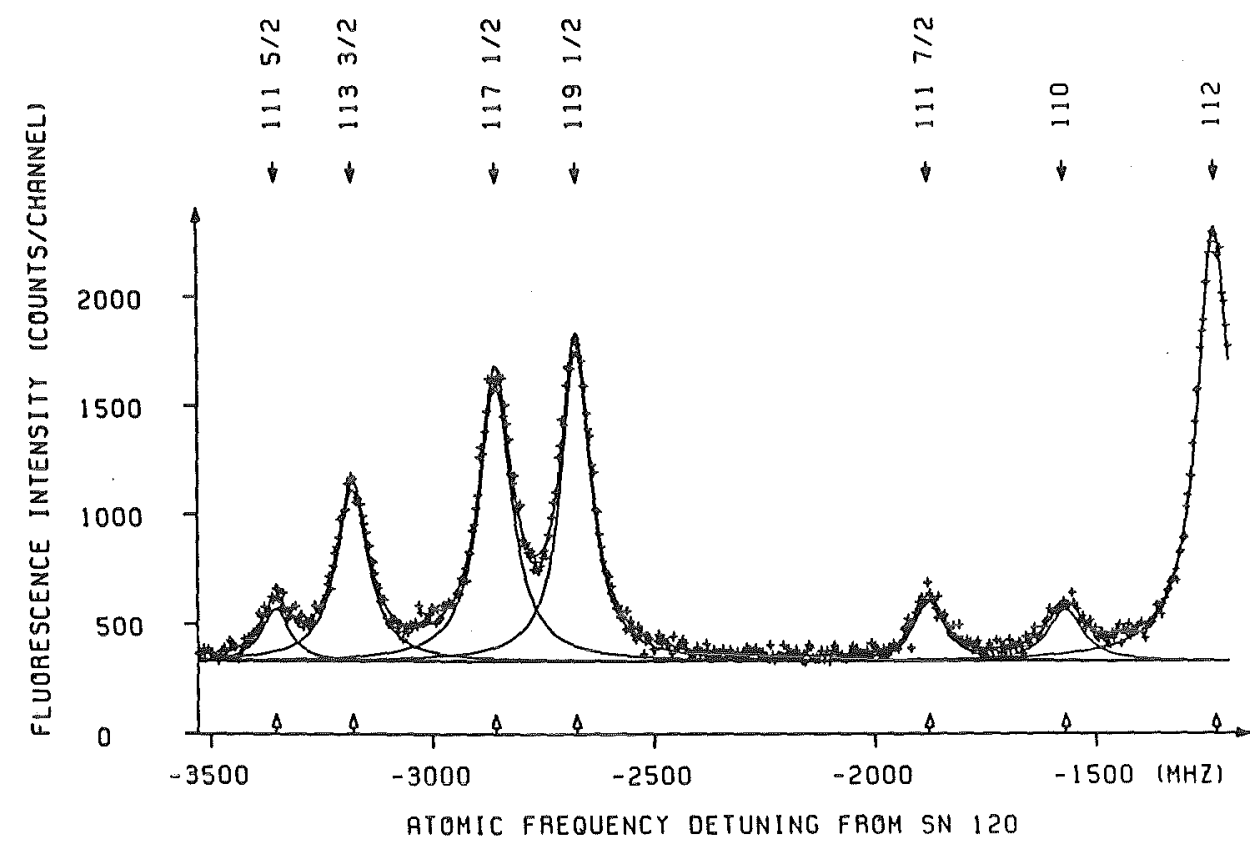

FIG. 2. Scan result for ${ }^{111} \mathrm{Sn} ; 113,110_{\mathrm{Sn}}$ and most of the ${ }^{112} \mathrm{Sn}$ are present since they are produced along with ${ }^{111} \mathrm{Sn} .117,119 \mathrm{Sn}$ are impurities.

The digital records were evaluated fitting them by a Lorentzian profile. Sometimes a correction was required if a strong resonance of another isotope was close by, e.g. ${ }^{114} \mathrm{Sn}$ and ${ }^{115} \mathrm{Sn}(\mathrm{F}=3 / 2)$ are obscured by ${ }^{116} \mathrm{Sn}$ and ${ }^{117} \mathrm{Sn}(F=3 / 2)$, respectively. To check the corrections, separate measurements were made with enriched samples for some but not all of the less abundant isotopes. This test showed that the correction is sufficiently accurate for the present purpose. There is, nevertheless, some remaining uncertainty as to the size of the correction since it depends on the line shape of the perturbing resonance far off the center. The actual lineshape in the wings was not determined to high accuracy; in addition, it changed slightly with the collimation and the oven position. It is assumed, however, that the errors due to the use of a pure Lorentzian can be ignored. The component positions found in this 
way are summarized in Table 1; ${ }^{120} \mathrm{Sn}$ is used as reference isotope although for technical reasons in many cases the actual experiment was performed with some other reference isotope. The errors quoted are not the bare statistical ones as they result from the fits; these are quite small and would mostly be an underestimate of the true error. The uncertainties result from a detailed discussion of the actual experiment. For the group of experiments with radioactive samples the main error stems from crucible motions which result in Doppler shifts. This is particularly true for those cases where the crucible was replaced to measure the stable reference isotope. A resonance was reproducible only to within $\pm 3 \mathrm{MHz}$ under exchange of a crucible. The corresponding reproducibility of the crucible position is $\pm 200 \mu \mathrm{m}$. In addition the fast temperature changes as the cold crucible is inserted and thereafter rapidly heated up causes two further errors: A crucible motion combined with a fast variation of the atomic beam intensity during the period of a single scan. Where these effects were important, the final error was further increased accordingly.

For the isotopes $117 \mathrm{~m}, 121,125 \mathrm{Sn}$ one hyperfine component almost coincides with components of the stable isotopes $115,119,119 \mathrm{Sn}$, respectivily, which might be present as an impurity with only poorly known quantity in the radioactive sample. A detailed analysis of the combined signals allowed us to give the component position to within reasonable error limits.

A special problem was the component $F=9 / 2$ of ${ }^{111} \mathrm{Sn}$. It could not be observed because of target contamination with natural $\mathrm{Sn}$ as mentioned above. The position of this component was estimated as follows: Assuming that $B$ is 0 or very small as for the corresponding isotopes $121,123,125 \mathrm{Sn}$, the $\mathrm{F}=9 / 2$ component lies close to the center of the strong ${ }^{120} \mathrm{Sn}$ line. If this is true, the ${ }^{111} \mathrm{Sn}$ component indeed is completely masked by ${ }^{120} \mathrm{Sn}$. As a conservative guess of $\mathrm{Q}$ we therefore take $Q=0(0.12)$ barn, which is reasonable in view of the $Q$ values for $121,123,125 \mathrm{Sn}$. The component position then is $+20(40) \mathrm{MHz}$. This preliminary result is subject to further investigations aiming at a target handling procedure that reduces the contamination by stable Sn.

As to the stable isotopes, the situation is quite different. They were finally measured under the best conditions achieved, i.e. without crucible or temperature changes. The single resonance results then scattered up to $500 \mathrm{kHz}$. To determine a spacing, the two resonance 
TABLE I. Hyperfine component positions and the resulting isotope shift for tin isotopes and isomers together with the rms charge radius changes and the staggering parameters as derived thereof. ${ }^{120} \mathrm{Sn}$ was chosen as reference. F designates the total angular momentum quantum number of the atom in the upper electronic state.

\begin{tabular}{|c|c|c|c|c|c|c|}
\hline $\begin{array}{l}\text { Mass } \\
\text { Number } \\
\text { A }\end{array}$ & $\begin{array}{l}\text { Nuclear } \\
\text { Spin } \\
\text { I }\end{array}$ & $\begin{array}{l}\text { Total } \\
\text { Angular } \\
\text { Momentum } \\
\quad \mathrm{F}\end{array}$ & $\begin{array}{l}\text { Component } \\
\text { position } \\
\text { [MHz] }\end{array}$ & $\begin{array}{l}\text { Isotope } \\
\text { Shift } \\
\text { [MHz] }\end{array}$ & $\begin{array}{c}\text { rms charge } \\
\text { radius changes } \\
\delta\left\langle\mathrm{r}^{2}\right\rangle \\
\mathrm{A}, 120 \\
{\left[\mathrm{fm}^{2}\right]}\end{array}$ & $\begin{array}{c}\text { staggering } \\
\text { parameter } \\
\gamma\end{array}$ \\
\hline 110 & 0 & 1 & $-1566.5(7.0)$ & $-1566.5(7.0)$ & $-0.638(20)$ & - \\
\hline 111 & $7 / 2$ & $\begin{array}{l}5 / 2 \\
7 / 2 \\
9 / 2\end{array}$ & $\begin{array}{r}-3351(10) \\
-1876(10) \\
20(40)\end{array}$ & $-1455(17)$ & $-0.586(19)$ & $0.67(13)$ \\
\hline 112 & 0 & 1 & $-1218.29(15)$ & $-1218.29(15)$ & $-0.497(15)$ & - \\
\hline 113 & $1 / 2$ & $\begin{array}{l}1 / 2 \\
3 / 2\end{array}$ & $\begin{array}{l}+3124.9(3.0) \\
-3181.4(3.0)\end{array}$ & $-1079.3(2.3)$ & $-0.438(14)$ & $0.91(3)$ \\
\hline 114 & 0 & 1 & $-900.68(15)$ & $-900.68(15)$ & $-0.367(11)$ & - \\
\hline 115 & $1 / 2$ & $\begin{array}{l}1 / 2 \\
3 / 2\end{array}$ & $\begin{array}{l}+3589.24(15) \\
-3002.00(15)\end{array}$ & $-804.92(12)$ & $-0.322(10)$ & $0.68(6)$ \\
\hline 116 & 0 & 1 & $-576.04(15)$ & $-576.04(15)$ & $-0.236(7)$ & - \\
\hline 117 & $1 / 2$ & $\begin{array}{l}1 / 2 \\
3 / 2\end{array}$ & $\begin{array}{l}+4314.02(20) \\
-2864.16(15)\end{array}$ & $-471.43(12)$ & $-0.189(6)$ & $0.75(6)$ \\
\hline $117 \mathrm{~m}$ & $11 / 2$ & $\begin{array}{l}9 / 2 \\
11 / 2 \\
13 /\end{array}$ & $\begin{array}{l}+3482.2(4.0) \\
+\quad 89.5(2.0) \\
-3806.7(3.0)\end{array}$ & $-483.3(1.8)$ & $-0.192(6)$ & \\
\hline 118 & 0 & 1 & $-270.24(15)$ & $-270.24(15)$ & $-0.112(4)$ & - \\
\hline 119 & $1 / 2$ & $\begin{array}{l}1 / 2 \\
3 / 2\end{array}$ & $\begin{array}{l}+4828.36(20) \\
-2683.57(15)\end{array}$ & $-179.59(12)$ & $-0.070(2)$ & $0.75(5)$ \\
\hline 120 & 0 & 1 & 0 & 0 & 0 & 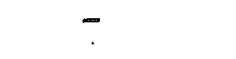 \\
\hline 121 & $3 / 2$ & $\begin{array}{l}1 / 2 \\
3 / 2 \\
5 / 2\end{array}$ & $\begin{array}{r}-2687.3(3.0) \\
-1023.9(3.0) \\
1760.0(5.0)\end{array}$ & $+90.8(2.7)$ & $+0.042(2)$ & $0.83(4)$ \\
\hline $121 \mathrm{~m}$ & $11 / 2$ & $\begin{array}{r}9 / 2 \\
11 / 2 \\
13 / 2\end{array}$ & $\begin{array}{l}+4000.9(3.0) \\
+663.8(3.0) \\
-3241.7(3.0)\end{array}$ & $+72.0(1.7)$ & $+0.037(2)$ & \\
\hline 122 & 0 & 1 & $+235.35(15)$ & $+235.35(15)$ & $+0.101(4)$ & - \\
\hline 123 & $11 / 2$ & $\begin{array}{r}9 / 2 \\
11 / 2 \\
13 / 2\end{array}$ & $\begin{array}{l}+4167.2(3.0) \\
+895.0(4.0) \\
-2979.4(3.0)\end{array}$ & $+297.2(2.0)$ & $+0.134(6)$ & $0.71(7)$ \\
\hline 124 & 0 & 1. & $+441.15(15)$ & $+441.15(15)$ & $+0.192(7)$ & - \\
\hline 125 & $11 / 2$ & $\begin{array}{r}9 / 2 \\
11 / 2 \\
13 / 2\end{array}$ & $\begin{array}{l}+4308 \cdot 5(4.0) \\
+1098(20) \\
-2722(8)\end{array}$ & $+504.3(7.5)$ & $+0.225(9)$ & \\
\hline
\end{tabular}


positions were measusured many times in turn with a period of about one minute. The resulting spacings had a short term statistical scatter of only $50 \mathrm{kHz}$ or less. The long term reproducibility, however, was worse; all independent results lie within an interval of $\pm 100 \mathrm{kHz}$ but are sometimes statistically not consistent. We therefore quote in these cases a conservative error of $\pm 150 \mathrm{kHz}$ which is $\pm 0.3 \%$ of the observed linewidth. The problems associated with measurements to such small fractions of the linewidth and the non-statistical errors of unknown origin found in several other examples will be discussed in a forthcoming paper. ${ }^{30}$

For the stable tin isotopes the present optical transition has been measured before with similar methods. ${ }^{22}$ Some of the results are inconsistent with ours; this triggered a detailed investigation of possible systematic errors which led to the accuracy as described in the preceeding paragraph. Among these studies are variations of the light power, the direction of the atomic and laser beams, the radio frequency used, the sideband combinations and the dye laser feedback circuit. The tests showed a reproducibility well within the $150 \mathrm{kHz}$ limit given above. In addition to this internal consistency check we have performed the same experiments with ${ }^{57} \mathrm{Co}$; the test included the determination of well known hyperfine splittings which were reproduced to within $\pm 50 \mathrm{kHz}$. The details will be given elsewhere. ${ }^{30}$ it should be noted, however, that the discrepancies with Ref. 22 are far beyond the range where they are relevant with respect to the nuclear quantities to be extracted.

\section{$\underline{V}$. Results and discussion}

From the results of the measurements given in Table 1 isotopic and isomeric shifts as well as the values of the hyperfine structure constants (see Table II) have been derived. The experimental quantities are then evaluated in terms of nuclear charge radius differences and nuclear electromagnetic moments, respectively. Using the standard relations between the isotope shift $\delta \nu_{A^{\prime}, A}=\nu_{A^{\prime}}-\nu_{A}$ and the mean square charge radius difference $\delta\left\langle r^{2}\right\rangle A^{\prime}, A=\left\langle r^{2}\right\rangle A^{\prime}-\left\langle r^{2}\right\rangle$ :

$$
\delta v_{A^{\prime}, A}=F \cdot \delta\left\langle r^{2}\right\rangle A^{\prime}, A+M \cdot\left(A^{\prime}-A\right) /\left(A^{\prime} \cdot A\right)
$$

we follow the procedure of Ref. 22 for determining the electronic parameters $F$ and $M$. It is based on the result $\delta\left\langle r^{2}\right\rangle{ }_{124,116}=+0.428(14) \mathrm{fm}^{2}$ (as obtained from the isotope shift of the $K_{\alpha}$ transition $^{32}$ ) combined with isotope shift and hyperfine splitting results for $\lambda=645 \mathrm{~nm}$ of $\mathrm{Sn} 11$. The 
TABLE II. Hyperfine splitting factors A and B with the corresponding nuclear moments $\mu_{I}$ and $Q$ for tin isotopes and isomers. The values marked with * are taken from Ref. 33 and 34. ** This is an assumption.

\begin{tabular}{|c|c|c|c|c|}
\hline $\begin{array}{c}\text { Mass } \\
\text { Number } \\
\text { [A] }\end{array}$ & $\begin{array}{c}\mathrm{A} \\
\text { Factor } \\
{[\mathrm{MHz}]}\end{array}$ & $\begin{array}{l}\text { B } \\
\text { Factor } \\
{[\mathrm{MHz}]}\end{array}$ & $\begin{array}{c}\mu_{I} \\
{\left[\mu_{N}\right]}\end{array}$ & $\begin{array}{c}Q_{S} \\
\text { [barn] }\end{array}$ \\
\hline 111 & $+421.5(5.6)$ & $0(19)$ & $+0.617(8)$ & $0(0.12)^{* \star}$ \\
\hline 113 & $-4204.2(2.8)$ & - & $-0.8791(6)$ & - \\
\hline 115 & $-4394.16(14)$ & - & $-0.91883(7)^{*}$ & - \\
\hline 117 & $-4785.45(17)$ & - & $-1.00104(7)^{*}$ & - \\
\hline $117 \mathrm{~m}$ & $-606.7(4)$ & $+53.3(3.3)$ & $-1.3955(10)$ & $-0.42(5)$ \\
\hline 119 & $-5007.95(17)$ & - & $-1.04728(7) *$ & - \\
\hline 121 & $+1112.4(1.7)$ & $+2.3(2.1)$ & $+0.6978(10)$ & $-0.02(2)$ \\
\hline $121 \mathrm{~m}$ & $-603.3(4)$ & $+18(4)$ & $-1.3877(9)$ & $-0.14(3)$ \\
\hline 123 & $-595.6(4)$ & $-3.4(4.6)$ & $-1.3700(9)$ & $+0.03(4)$ \\
\hline 125 & $-586.0(9)$ & $-12(21)$ & $-1.348(2)$ & $+0.09(17)$ \\
\hline
\end{tabular}


above expression may be rewritten more specifically for the observed isotope shift of the $\lambda=286 \mathrm{~nm}$ transition

$$
\begin{aligned}
& \left\langle r^{2}\right\rangle-\left\langle r^{2}\right\rangle=\delta\left\langle r^{2}\right\rangle_{A^{\prime}, A}=\frac{A^{\prime}-A}{A^{\prime} \cdot A} \cdot \frac{124 \cdot 116}{124-116} \cdot 0.428(14) \mathrm{fm}^{2} \\
& +\frac{1}{3.3(0.3) \mathrm{GHz}} \cdot\left\{\left(\nu_{A^{\prime}}-v_{A}\right)^{2}-\frac{A^{\prime}-A}{A^{\prime} \cdot A} \cdot \frac{124 \cdot 116}{124-116} \cdot\left(\nu-\nu_{124}\right)\right\}
\end{aligned}
$$

The resulting $\delta\left\langle r^{2}\right\rangle$ values are given in Table $I$ and their variation with $A$ is displayed in Fig. 3. The quoted errors mainly arise from the uncertainty of the calibration value $\delta\left\langle r^{2}\right\rangle_{124,116}$. Fig. 3 compares with the naive liquid drop estimate $\left(\left\langle r^{2}\right\rangle_{A}=3 / 5 \cdot r_{0}^{2} \cdot A^{2 / 3}\right.$ with $\left.r_{0}=1.2 \mathrm{fm}\right)$ and the prediction of the (spherical) droplet model ${ }^{33} 34$, neglecting contributions from the variation of the mean square deformation $\left\langle\beta^{2}\right\rangle$ (or of the skin thickness of the nuclear charge distribution).

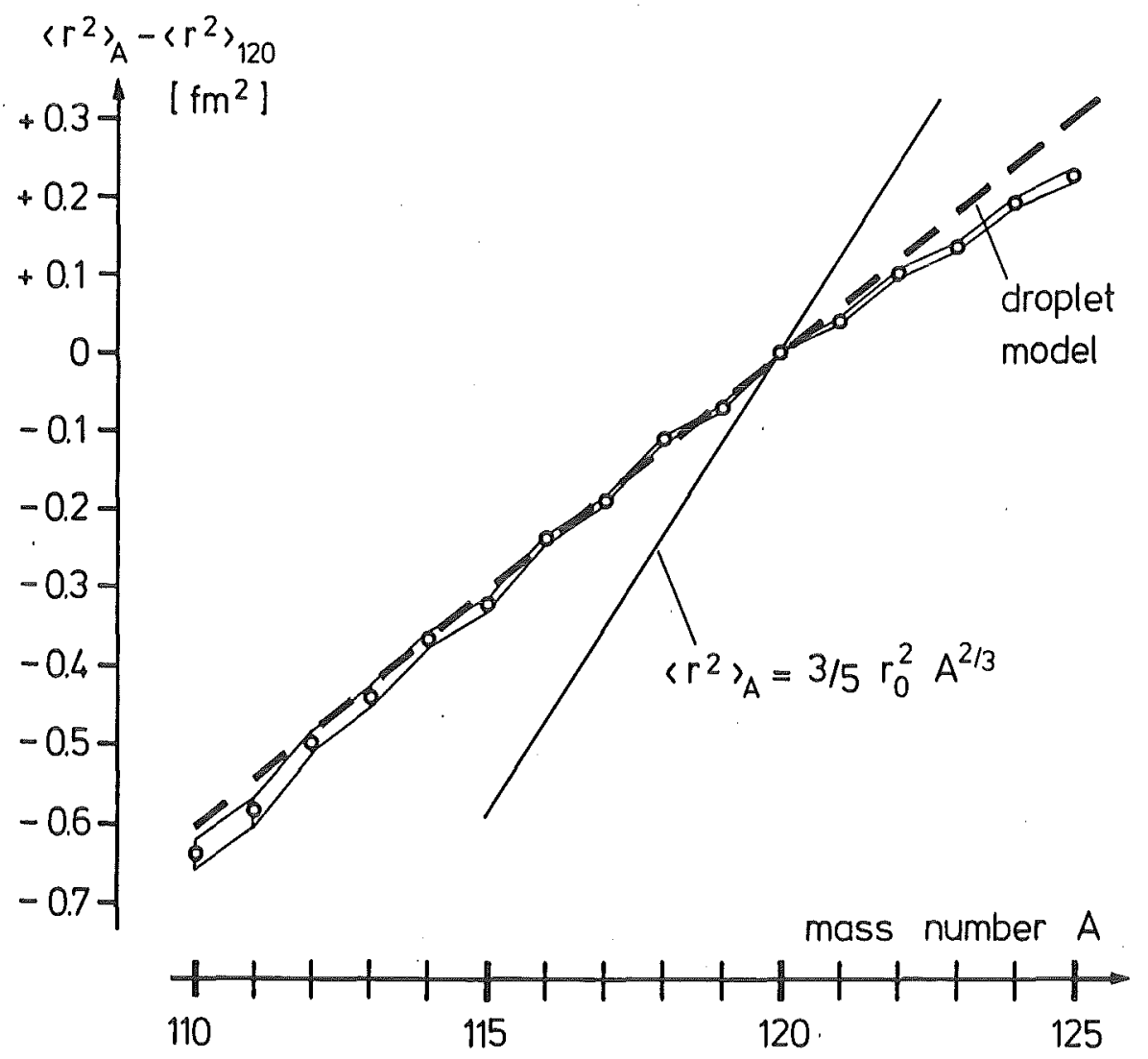

FIG. 3. The variation of the mean square charge radii of $\mathrm{Sn}$ nuclei with the mass number $A$ as compared to the droplet model prediction. The systematic uncertainty arising from the calibration of the experimental data is indicated by an "error band" enclosing the data points. 
For an estimate of the $\left\langle\beta^{2}\right\rangle$ influence, the variation of the mean square deformation is derived (within some systematic uncertainty) from the electromagnetic transition probabilities. The consideration of the $B\left(E 2 ; 0^{+} \rightarrow 2+\right.$ ) values of the even $S n$ nuclei leads to the conclusion that $\left\langle\beta^{2}\right\rangle$ is slightly increasing with decreasing neutron numbers. ${ }^{20}$ An explicit account for such a variation would lift the droplet model curve for smaller $A$ and deteriorate the good overall agreement between the observed data and the spherical droplet model. This feature is similarly observed for neutron deficient $\mathrm{Cd}$ nuclei; ${ }^{26}$ it may indicate that deformation effects play a minor role as compared to other nuclear structure effects.

In order to reveal the finer details, Fig. 4 displays the $\left\langle r^{2}\right\rangle$ changes for isotopes that differ by two units on the mass scale $\left(\delta<r^{2}>A, A-2\right)$. The advantage of such a differential plot (Brix-Kopfermann plot) is that the uncertainty is smaller since $\delta A$ is small. $\delta A=2$ is chosen in order to eliminate the odd-even staggering that dominates the changes with $\delta A=1$. The droplet model predicts a constant increase of $\delta\left\langle r^{2}\right\rangle$ with $A$.

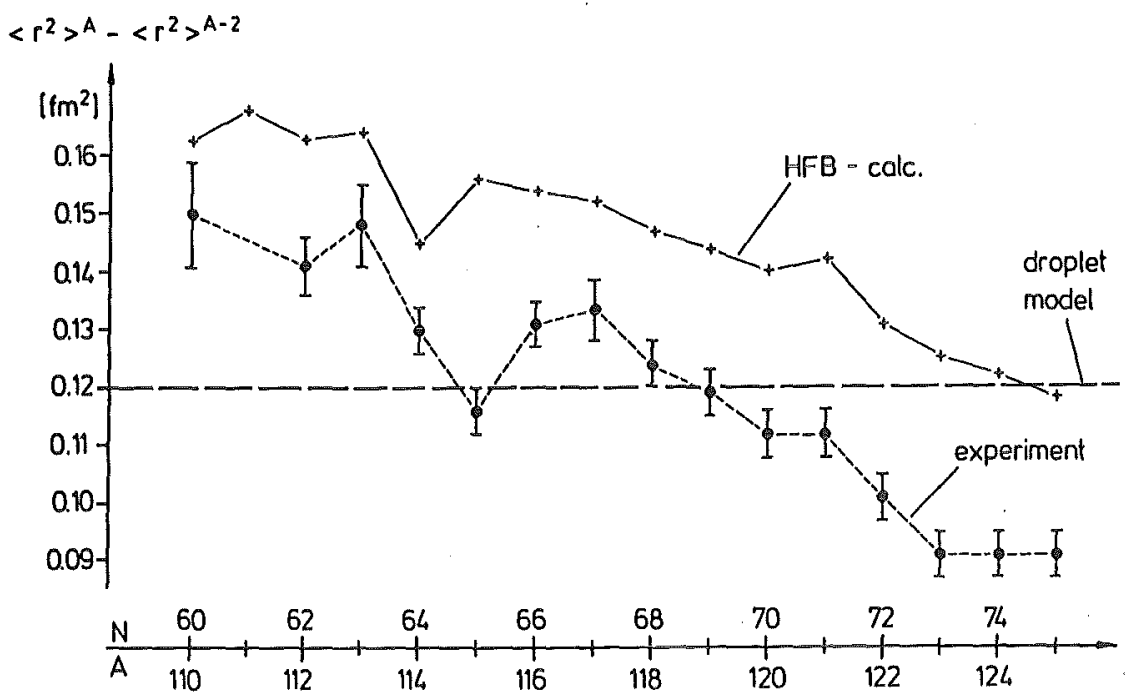

FIG. 4. The differential change $\delta\left\langle r^{2}\right\rangle, A, A-2$ of the mean square charge radii of $\mathrm{Sn}$ compared with theoretical results on the basis of the HFB theory. ${ }^{11}$ The data for $N=60\left(108,110_{S n}\right)$ were taken from Ref. 20.

The experimental results show a distinct drop at $N=64$ which may be attributed to the subshell closure when going from the $d_{5 / 2}, g_{7 / 2}$ to the $s_{1 / 2}, d_{3 / 2}$ and $h_{11 / 2}$ neutron orbits. This feature has already been shown in connection with studies of the $\mathrm{ms}$ radii of stable even $\mathrm{Cd}$ 
isotopes. ${ }^{26}$ In fact, microscopic nuclear structure calculations ${ }^{1 / 1}$ on the basis of the Hartree-Fock-Bogolyubov (HFB) theory do predict such a dip, however shifted by one unit on the mass scale. This discrepancy is unexplained at present.

The finest detail in the observed variation of $\left\langle r^{2}\right\rangle$ is the distinct oddeven staggering. This is traditionally represented by the parameter $\gamma$ defined as:

$$
\gamma=2 \cdot\left(\left\langle r^{2}\right\rangle_{A}-\left\langle r^{2}\right\rangle_{A-1}\right) /\left(\left\langle r^{2}\right\rangle_{A+1}-\left\langle r^{2}\right\rangle_{A-1}\right)
$$

which can be expressed more directly by the more accurate shifts $\delta \nu_{A}$, A

$$
\gamma=2 \cdot \frac{{ }^{\delta \nu_{A}}, A-1-M /(A \cdot(A-1))}{\delta \nu_{A}+1, A-1-2 \cdot M /((A+1) \cdot(A-1))}
$$

thus eliminating the electronic parameter $F$ and its uncertainty. The deviation of $\gamma$ from unity is a measure for the degree and direction of the actual odd-even staggering. The values calculated with $\mathrm{M}=671(480) \mathrm{GHz}$ (taken from Ref. 22) are listed in Table 1.

For some considerations an absolute measure of the staggering is more appropriate, ${ }^{35}$ namely the quantity

$$
\begin{aligned}
\Delta & =\left\langle r^{2}\right\rangle_{A}-\frac{1}{2} \oplus\left(\left\langle r^{2}\right\rangle_{A-1}+\left\langle r^{2}\right\rangle+1\right) \\
& \left.=\frac{1}{2} \cdot\left(\delta<r^{2}\right\rangle_{A, A-1}-\delta\left\langle r^{2}\right\rangle_{A, A+1}\right)
\end{aligned}
$$

which is plotted in Fig.5; it exhibits the different behaviour of the even and odd $\mathrm{Sn}$ isotopes quite clearly.

For the odd isotopes and the isomers the splitting constants $A$ and $B$ for the magnetic dipole and electric quadrupole hyperfine interaction have been determined. They are given in Table II. From $A$ the nuclear magnetic dipole moments $\mu_{1}$ were calculated using the well known magnetic moments of $115,117,119 \mathrm{Sn} .{ }^{36,37}$ The errors given for the unstable isotopes do not include a possible hyperfine anomaly since it is below $5 \cdot 10^{-5}$ for the odd stable isotopes as results from a comparison with a precise $g_{1}$ ratio determination ${ }^{38}$. The same has been found in two states of another electron configuration of tin. ${ }^{23 / 38}$ 
The magnetic moments reflect mainly the single particle properties of the ground and isomeric states investigated. The observed values are consistent with the Schmidt values. The deviations from the Schmidt values (actually up to $50 \%$ ) may orginate from core polarization and mesonic effects.

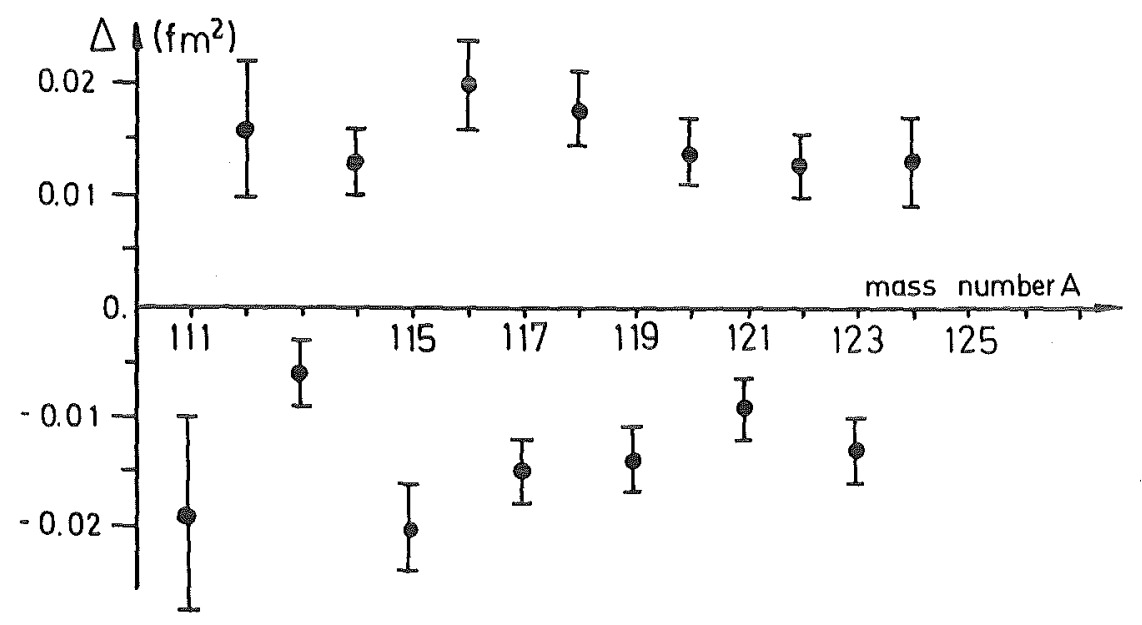

FIG. 5. The odd-even staggering of the mean square charge radii of $\mathrm{Sn}$ nuclei as represented by the quantity $\Delta$ as defined by equation (5).

The extraction of the quadrupole moments from the observed $B$ factors requires the value of the electric field gradient produced by the atomic electrons at the nuclear site or of the quantity $\left\langle r^{-3}\right\rangle_{5 p}$. A rough estimate can be obtained from the spin orbit splitting of the $5 p 6 s$ configuration. With the energy level data as taken from ${ }^{27}$ the spin orbit interaction constant from standard formulae 39,40 results to be $\zeta=2659 \mathrm{~cm}^{-1}$. It follows $\left.{ }^{4}<^{-3}\right\rangle_{5 p}=9.45 \cdot a_{0}{ }^{-3}$. A more refined value has been calculated by Dembczynski and Rebel $^{42}$ using a semiempirical approach. Their result in terms of effective operator radial parameters is $\left.<^{-3}\right\rangle_{5 p}^{02}=10.2(6) \cdot a_{0}{ }^{-3}$. This value does not include sternheimer core polarization type effects. With the latter $\left\langle r^{-3}\right\rangle_{5 p}^{02}$ it follows $\mathrm{Q}_{\mathrm{s}}=-0.0078(5) \cdot \mathrm{B}\left(5 \mathrm{p} 6 \mathrm{~s} ;{ }^{3} \mathrm{P}_{1}\right)$ barn $/ \mathrm{MHz}$. The $\mathrm{Q}_{\mathrm{s}}$ values given in Table II are calculated with this relation.

A discussion of the spectroscopic quadrupole moments in terms of the intrinsic nuclear deformation, by relating $Q_{s}$ to the experimentally observed $B(E 2)$ values or values of the $m s$ deformation, respectively, is highly model dependent. In the case of the $S n$ nuclei with $\left\langle\beta^{2}\right\rangle^{1 / 2} \sim 0.1$ 
the usual "strong coupling" relation (though sometimes applied in such cases) is hardly a basis of a meaningful comparison. The larger value of $Q_{S}$ of the $11 / 2$ isomer $117 m_{S n}$ as compared to the values of neighbouring Sn nuclei must be considered together with a rather small isomer shift $\delta\left\langle r^{2}\right\rangle 117 \mathrm{~m}, 117$, thus indicating a rather complex relation between $Q_{s}$ and the intrinsic nuclear deformation.

\section{Concluding remarks}

The low-lying energy level spectra of the $\mathrm{Sn}$ nuclei indicate a coexistence of states of distinct rotational character with vibrational structures. This interesting feature is common with other nuclei with proton or neutron numbers near a closed shell value. Generalized collective models like the Gneuß-Greiner model ${ }^{43}$ or refined interacting boson models (IBM) 44,45 attempt to account for such mixed situations and provide the framework for a coherent discusssion of the experimental data: The level schemes, the transition probabilities, the electromagnetic moments and the nuclear radii as well. The nuclear structure information specifically provided by the variation of the nuclear charge radii has recently been analyzed by Alonso, Arias and lachello46/47 on the basis of the IBM-244 and IBFM-2 $2^{45}$ models, showing interesting nuclear structure aspects of the nuclear radius variation.

The main result of our present investigations is a set of precise experimental data on stable and radioactive $S n$ nuclides, which may serve as basis of a detailed theoretical discussion in the framework of an advanced nuclear structure model. The observed trend in the Sn charge radii seems to be less affected by deformation effects (this is supported by the experimental quadrupole moments), however we observed irregularities associated with more detailed shell effects (subshell closure at $N=64$ ) or a distinct odd-even staggering for which a quantitative microscopic explanation is missing up to now.

We acknowledge the interest and valuable communications of $\mathrm{J}$. Dembczynski, G. Huber and I. Talmi and thank B. Feurer for his technical assistance during the measurements. 


\section{References}

${ }^{1}$ D.N. Stacey, Rep. Prog. Phys. 29, 171 (1966).

${ }^{2}$ B. Hoffmann, G. Baur, and J. Speth, Z. Phys. A 320, 259 (1985).

${ }^{3}$ P. Jacquinot and R. Klapisch, Rep. Prog. Phys. 42, 773 (1979).

${ }^{4} E .-W$. Otten, 4th Internat. Conference on Nuclei far from Stability, Helsing $\phi r$, CERN 81-09, p. 3 (1981).

${ }^{5}$ Proc. Conf. on Lasers in Nuclear Physics, Oak Ridge Tenn., April 21-23 1982. Eds. C.E. Bemis Jr. and H.K. Carter Harwood Academic

Publ. Chur-London-New York 1982.

${ }^{6}$ R.C. Thompson, Rep. Prog. Phys. 48, 531 (1985).

${ }^{7}$ W.D. Myers and W.T. Swiatecki, Ann. Phys. 55, 395 (1969).

${ }^{8}$ E. Wesolowski, J. Phys. G: Nucl. Phys. 11, 909 (1985).

${ }^{9} \mathrm{D}$. Berdichevsky and $\mathrm{F}$. Tondeur, Z. Phys. A 322, 141 (1985).

$10 \mathrm{~J}$. B. McGrory and B.A. Brown, ibid. 5, p. 455.

$11 \mathrm{~J}$. Dobaczewski, M. Flocard, and J. Treiner, Nucl. Phys. A422, 103 (1984).

121. Talmi, Nucl. Phys. A4234, 189 (1984).

${ }^{13}$ D. Zawischa, Phys. Lett. 155 B, 309 (1985).

${ }^{14}$ W.D. Myers and K. Schmidt, Nucl. Phys. A410, 61 (1983).

${ }^{15} \mathrm{H}$. Rebel, Proc. $X X$. Winter School on Physics: Selected Topics of Nuclear Structure, April 13-19, 1985, Zakopane, Poland.

Eds. J. Styczen and R. Broda.

${ }^{26} \mathrm{C}$. Thibault, F. Touchard, S. Büttgenbach, R. Klapisch, M. de Saint Simon, H.T. Duong, P. Jacquinot, P. Juncar, S. Liberman, P. Pillet, J. Pinard, J.L. Vialle, A. Pesnelle, and G. Huber, Phys. Rev. C 23, 2720 (1981).

${ }^{17}$ R.C. Thompson, M. Anselment, K.Bekk, S.Göring, A. Hanser, G. Meisel, H. Rebel, G. Schatz, and B.A. Brown, J. Phys. G: Nucl, Phys. 9, 443 (1983).

${ }^{18} \mathrm{M}$. Anselment, W. Faubel, S.Göring, A. Hanser, G. Meisel, H. Rebel, and G. Schatz, Nucl. Phys. A451, 471 (1986).

${ }^{19} \mathrm{M}$. Anselment, Thesis, Universität Heidelberg (1984) and KfK Report 3797 (1984), ISSN 0303-4003.

${ }^{20} \mathrm{H}$. Lochmann, Thesis, Universität Mainz (1985) and GSI Report 85-8 (1985), ISSN 0171-4546.

${ }^{21}$ J.D. Silver and D.N.Stacey, Proc. R. Soc. London A332, 139 (1973); ibid. A332, 129 (1973).

${ }^{22}$ P.E.G.Baird, S. Blundell, G. Burrows, C.J. Foot, G. Meisel, D.N. Stacey, and G.K. Woodgate, J. Phys. B16, 2485 (1983). 
${ }^{23}$ W.J. Childs, Phys. Rev. A4, 439 (1971).

${ }^{24}$ M.H. Prior, A. Dymanns, H.A. Shugart, P.A. Van den Bont, Phys. Rev. 181, 1665 (1969).

${ }^{25}$ A. Andl, K. Bekk, S. Göring, A. Hanser, G. Nowicki, H. Rebel, G. Schatz, and R.C. Thompson, Phys. Rev. C26, 2194 (1982).

${ }^{26}$ R. Wenz, A. Timmermann, and E. Matthias, Z. Physik A303, 87 (1981) and references therein.

${ }^{27}$ C.E. Moore, Atomic Energy Levels, Circ. Natl. Bur. Std. (US) 467, Sect. III (1958).

${ }^{28}$ W.F. Meggers, C.H. Corliss, and B.F. Scribner, Tab. Spectral Line Intensities, Natl. Bur. Std. (US) 145/I (1975).

${ }^{29}$ B. Burghardt, W. Jitschin, and G. Meisel, Appl. Phys. 20, 141 (1979). G. Meisel, Laser und Optoelectronik, 15, 105 (1983); ibid. 15, 245 (1983).

${ }^{30} \mathrm{M}$. Anselment and $\mathrm{G}$. Meisel, to be published.

${ }^{3} \mathrm{M}$. Anselment, S. Chongkum, H. Hoeffgen, and G. Meisel, KfK Report 3621,65 (1983), ISSN 0303-4003.

${ }^{32}$ S. Bhattacherjee, F. Boehm, and P.L. Lee, Phys. Rev. 188, 1919 (1969).

${ }^{3}$ W.D.Myers, Droplet Model of the Nucleus (IFI/Plenum Data Co, New York, 1977).

${ }^{34}$ W.D. Myers, ibid. ${ }^{5}$, p. 437.

${ }^{35} \mathrm{I}$. Talmi, private communication.

${ }^{36} \mathrm{~W}$. Proctor, Phys. Rev. 79,35 (1950).

${ }^{37}$ C.M. Lederer and V.S.Shirley, Table of Isotopes. (John Wiley and Sons, New York 1978).

${ }^{38} \mathrm{H}$. Krüger, O. Lutz, A. Nolle, and A. UhI, Z. Naturforschung 27a, 173 (1973).

${ }^{39}$ E.U. Condon and G.H. Shortley, The Theory of Atomic Spectra (Cambridge: At the University Press, 1970).

${ }^{40}$ H.G. Kuhn, Atomic Spectra, 2nd ed. (Longmans, 1969).

${ }^{4}{ }^{1} \mathrm{H}$. Kopfermann, Kernmomente (Akademische Verlagsgesellschaft Frankfurt/M 1956).

4.2J. Dembczynski and H. Rebel, KfK-Report 3815, 64 (1984), ISSN $0303-4003$.

${ }^{43}$ G. Gneuß and W. Greiner, Nucl. Phys. A171, 449 (1971).

${ }^{44}$ A. Asima, T. Otsuka, F. lachello and I. Talmi, Phys. Lett. 66B, 205 (1977).

${ }^{45}$ N. Yoshida, A. Arima, and T. Otsuka, Phys. Lett. 114B, 86 (1982).

${ }^{46} \mathrm{C}$. E. Alonso, J.M. Arias, and F. lachello, Phys. Lett. 164B, 241 (1985).

${ }^{47} \mathrm{~F}$. lachello, Nucl. Phys. A358, 89c (1981). 